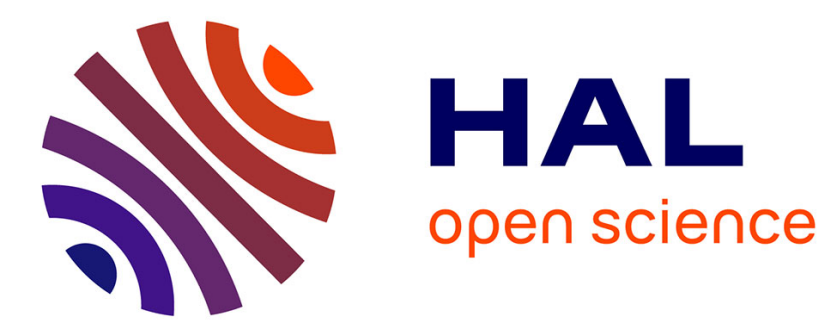

\title{
Heterogeneous melting of methane confined in nano-pores
}

E Dundar, P. Boulet, C Wexler, L Firlej, Ph Llewellyn, B Kuchta

\section{To cite this version:}

E Dundar, P. Boulet, C Wexler, L Firlej, Ph Llewellyn, et al.. Heterogeneous melting of methane confined in nano-pores. Journal of Chemical Physics, 2016, 145 (14), pp.144704. 10.1063/1.4964650 . hal-01477265

\section{HAL Id: hal-01477265 https://hal.science/hal-01477265}

Submitted on 27 Feb 2017

HAL is a multi-disciplinary open access archive for the deposit and dissemination of scientific research documents, whether they are published or not. The documents may come from teaching and research institutions in France or abroad, or from public or private research centers.
L'archive ouverte pluridisciplinaire HAL, est destinée au dépôt et à la diffusion de documents scientifiques de niveau recherche, publiés ou non, émanant des établissements d'enseignement et de recherche français ou étrangers, des laboratoires publics ou privés. 


\title{
Heterogeneous melting of methane confined in nano-pores
}

\author{
E. Dundar ${ }^{1, a)}$, P. Boulet ${ }^{1}$, C. Wexler ${ }^{2}$, L. Firlej ${ }^{3}$, Ph. Llewellyn $^{1}$, B. Kuchta ${ }^{1,4, b)}$ \\ ${ }^{1}$ Aix Marseille Université, CNRS, MADIREL (UMR 7246), 13397 Marseille, France \\ ${ }^{2}$ Department of Physics and Astronomy, University of Missouri, Columbia, MO 65211, USA \\ ${ }^{3}$ Laboratoire Charles Coulomb (L2C), UMR5221, Université de Montpellier, 34095 Montpellier, France \\ ${ }^{4}$ Department of Chemistry, Wroclaw University of Science and Technology, 50-370 Wroclaw, Poland
}

The melting transition of methane adsorbed in nanopores has been studied and compared in two types of structures: carbon slits pores and SURMOF square shaped channels. We show that the nano-confinement not only modifies the temperatures of phase transformation but also induces strong space heterogeneity of the adsorbate. We emphasize the role of the structural heterogeneity on the mechanism of melting: in nanometric pores, each adsorbed layer exhibits different mechanism of structural transformations and the notion of a unique transition temperature is not well defined.

\section{INTRODUCTION}

Since the discovery of nucleation reaction in the early $1930 \mathrm{~s}^{1-2}$, the influence of nanoscale structure on the thermodynamics properties of the nano-systems has been studied. The recent rapid progress in the synthesis and processing of materials with tunable nanometer scale structures has resulted in the need to define rigorously the thermodynamics for the nanoscale systems. In particular, the issue of characterization of structural transformations of such small objects is an area of intense investigation ${ }^{3-10}$. It is well known that the nanosystems exhibit properties significantly different from their bulk analogs. In particular, the position of coexistence lines depends on the size and shape of the nano-objects and the phase diagrams are redefined. This is a consequence of the fact that nanosystems are characterized by high surface-to-volume ratio; the surface atoms are weakly bonded and their contribution to the latent heat is smaller. Consequently, the surface usually transforms at lower temperature than the bulk and the whole transition may happen smoothly over a large (but finite) range of temperatures. This observation suggests that at the nanoscale there is no temperature of melting (or any other structural change) in the conventional sense: the structural (phase) changes are gradual and phases are no longer distinguishable.

a) Current email : Ege.Dundar@,vub.ac.be

b) Author to whom correspondence should be addressed. Electronic mail: : bogdan.kuchta@univ-amu.fr 
The nano-objects exhibit continued transformations because their small size naturally favors larger amplitude of fluctuations. In addition, the fluctuations' amplitude is location-dependent, due to the heterogeneity of any nano-system. Consequently, the overall behavior of the system is not uniform but space-dependent. The nanoobjects also exhibit some features typical for second order critical behavior; however, the inherent limitation of the correlation length results in a lack of a real critical behavior.

Nano-porous materials define a particular environment for studies of nano-objects' properties. Not only they naturally define the nanosize limits of the matter confined in the pores but they also introduce an additional external force field due to the interaction with the pore walls. This field is not uniform, it depends on the wall heterogeneity and its strength decreases with a distance from the walls. Therefore, the interactions within each layer of the confined system are different from each other and depend on the layer position. The competition between the external heterogeneous forces and the intermolecular interactions is a key factor that modifies the properties of any confined nano-system.

Atomistic numerical simulations offer a possibility to precisely account for heterogeneity and fluctuations in nanoporous system. The amplitude of the fluctuations depends on the system size and their evolution in space reflects directly the characteristics of the potential energy surface (PES) explored by the microscopic configurations during the simulations. In this way, the existence of metastable states is often revealed, even ones with short lifetimes that may be difficult to detect experimentally. This unique possibility is a consequence of the system size. Bulk systems may be trapped at low temperatures in a part of the configurational space due to the low probability of transitions into other equivalent volume of the configurational space. In nano-systems, the transitions between different configurations are easier due to the larger amplitude of fluctuations.

The structural transformations of methane confined in pores have been studied numerically for many years. Miyahara and Gubbins ${ }^{3}$ already gave the general analysis of methane melting in slit pores. The melting was studied in pores of different shape ${ }^{4-7}$; few examples of the solid-solid (hexagonal-tetragonal) transformations in slit pores were also reported ${ }^{6,7}$. In all cases, the common observed feature was a dependence of the transformation mechanism on the pores size. The strength of the adsorbate-adsorbent interaction was another important parameter defining the transition temperature ${ }^{8,9}$. 
In this paper, we emphasize the role of the structural heterogeneity of methane adsorbed in nanopores and we analyze its influence on the mechanism of structural transformations in such nano-confined system. Two particular situations are discussed: first, the melting transition of methane confined in 3 and $4 \mathrm{~nm}$ carbon slitshaped pores; second, the melting of methane confined in $2.8 \mathrm{~nm}$ pores formed by square channels of the SURMOF structure. The mechanism of melting in slit and square pores is then compared, and the correlation between the nano-dimension and heterogeneity and discussed in details. Finally, we discuss the simulated transformations in the context of the existing experimental information ${ }^{10}$.

\section{NUMERICAL METHODOLOGY}

The pore structures studied in this paper are shown in Fig.1. Two parallel graphene walls form the slitshaped carbon pores. We do not modeled multilayer graphene walls because their contribution is below $10 \%$ of the total interaction energy. We have studied two pore sizes: $\mathrm{z}=3 \mathrm{~nm}$ and $\mathrm{z}=4 \mathrm{~nm}$, where $\mathrm{z}$ is defined as the distance between the centers of the carbon atoms in the graphene sheets forming the pore. In the xy plane, the pores are virtually infinite and modeled using periodic boundary conditions. The xy box dimensions $(4.26 \mathrm{x} 4.92) \mathrm{nm}^{2}$ are commensurate with the graphene structure. However, the wall structure was homogeneous. The effect of the corrugation of graphene surface has been estimated to be very small compared to the mean interaction energy. In addition, the choice of homogeneous walls allows one to exclude any influence of the wall heterogeneity on the melting transformation. The SURMOF possesses one of the largest pores reported so far for MOFs. For the longest molecular linker (PPDC ${ }^{11}$ ), the size of the channel is $2.85 \times 2.85 \mathrm{~nm}^{2}$ and the structure has P4 symmetry. In our calculations, the channels have been modeled as infinitely long, again using periodic boundary conditions. The paper by Liu et al. ${ }^{11}$ have described the details of the SURMOF structure in the paper. The position of the atoms of the SURMOF structure used in our simulation are included in the supplementary material.

The computational modeling was based on grand canonical Monte Carlo (GCMC) simulations implemented in our own code, extensively tested and validated ${ }^{12-15}$. The carbon slit-shaped framework and SURMOF structure were assumed rigid during simulations. All interatomic interactions were modeled using the Lennard-Jones (6-12) potential. The $\mathrm{CH}_{4}$ molecules were considered as super-atoms (united atom model, UA). The $\mathrm{CH}_{4}-\mathrm{CH}_{4}$ Lennard-Jones parameters were $\sigma_{\mathrm{CH} 4}=3.730 \AA$ and $\varepsilon_{\mathrm{CH} 4}=148.0 \mathrm{~K}$. In slit pores, for methane-wall interactions, the Steele 10-4 potentials already tested in $^{16-17}$ were employed. Parameters describing fluid-solid 
interactions were obtained using the Lorentz-Berthelot mixing rules. The parameters for the framework atoms of SURMOF were obtained from the Universal Force Field (UFF). The interactions were cut-off beyond $15 \AA$. As the united atom representation of the $\mathrm{CH}_{4}$ molecule is isotropic, electrostatic forces were not considered in the simulations. Periodic boundary conditions were employed in the directions parallel to graphene walls for carbon slit-shape pores, and in all direction in SURMOF simulations. Adsorption isotherm points were calculated by averaging the number of adsorbed molecules per unit cell of the material for a given chemical potential $\mu(\mathrm{P})$ and temperature T. The ideal gas model was applied.
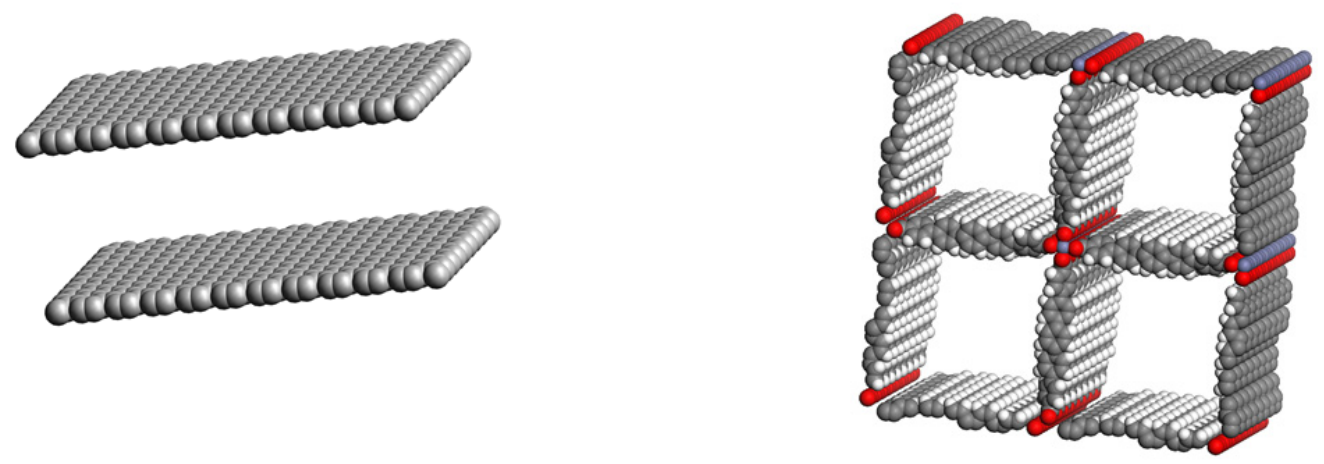

Fig.1. Slit pore (left) and SURMOF (right) structures. The hydrogen, carbon and oxygen atoms are represented by light grey, grey and red colors, respectively. In the simulation, the slit walls are homogeneous without any corrugation.

To characterize the mechanism of structural transformations within the adsorbed gas, we use two parameters: the $\Phi_{6}$ order parameter (to distinguish between the ordered solid-like structure and the disordered liquid layer) and the radial distribution function $\mathrm{g}(\mathrm{r})$. The $\Phi_{6}$ order parameter is defined as ${ }^{18}$

$$
\Phi_{6 j}=\mid\left\langle\frac{l}{N_{b}} \sum_{k=1}^{N_{b}} e^{i 6 \theta_{k}}\right\rangle
$$

and is able to characterize structural 6-fold symmetry of the center-of-mass lattice positions by measuring the average bond order within a plane layer. Here the azimuthal angle $\theta_{\mathrm{k}}$ defines an orientation of a vector ("bond") between centers of mass of each nearest-neighbor pair in a layer ${ }^{18}$. The index $\mathrm{k}$ runs over the total number of 
nearest-neighbor bonds $\mathrm{N}_{\mathrm{b}}$ in the adsorbed layer. $\Phi_{6}$ is equal to 1 if the structure represents ideal hexagonal (solid) order, and 0 if the structure is disordered (liquid). In this study, we analyze it separately for each adsorbed layer $\mathrm{j}$.

The second function used to characterize the state of the adsorbed layer is the radial distribution function $\mathrm{g}(\mathrm{r})$. The 2-dimensional version of $\mathrm{g}(\mathrm{r})$ are calculated inside each layer adsorbed in the slit pores. These correlations help to identify the nature of the various phases, from very ordered solid-like phase to more disordered (liquid crystal or liquid-like) phases.

\section{RESULTS}

\section{A. Adsorption mechanism and layering transition at $\mathrm{T}=90 \mathrm{~K}$.}

At $\mathrm{T}=90 \mathrm{~K}$ the adsorption of methane layers in slit pores is very regular because the adsorbent slit model has regular graphene-like walls. The isotherm of adsorption is step-wise (Fig. 2a); each subsequent layer is very stable and well ordered. However, any new layer formation is preceded by increased amplitude of structural and energy fluctuations, which indicate a destabilization of the adsorbed system before the new layer starts to form. In fact, for each layer, an optimal density exists that stabilizes the total adsorbed system. Such mechanism of layering transitions can be easily inferred from both the energy and the number of molecules fluctuations (Fig. 2b). The final filling of the pore (similar to capillary condensation) is also initiated by large fluctuations, especially, of the number of the adsorbed molecules. It is interesting to observe that the energy fluctuations show a global decreasing tendency with increased pressure. This is the direct consequence of the increasing contribution of the methanemethane interaction energy to the total energy of the adsorbed system. The final state of methane in the filled pore is characteristic for solid ordered arrangement in the contact layer. This is confirmed by the radial distribution function: the in-layer radial distribution function shows (Fig. 2c) that each layer has a different structure: the contact layer is solid, due to the ordering action of the strong interaction with the pore wall, whereas the third layer

is, at least partially, liquid. This heterogeneity of the adsorbed system is the key feature determining the mechanism of melting. It will be discussed below in the following part of this paper.

It is important to emphasize that the mechanism of melting in pores may be studied at any stage of pore filling, even in partially filled pores. This means that any particular mechanism of melting is always defined for particular thermodynamic path. In this paper, we analyze the melting in completely filled pore, that is, for 
thermodynamic conditions where the external pressures is higher than the pressure required for the last vertical jump observed on the isotherm curve (Fig. 2ad).
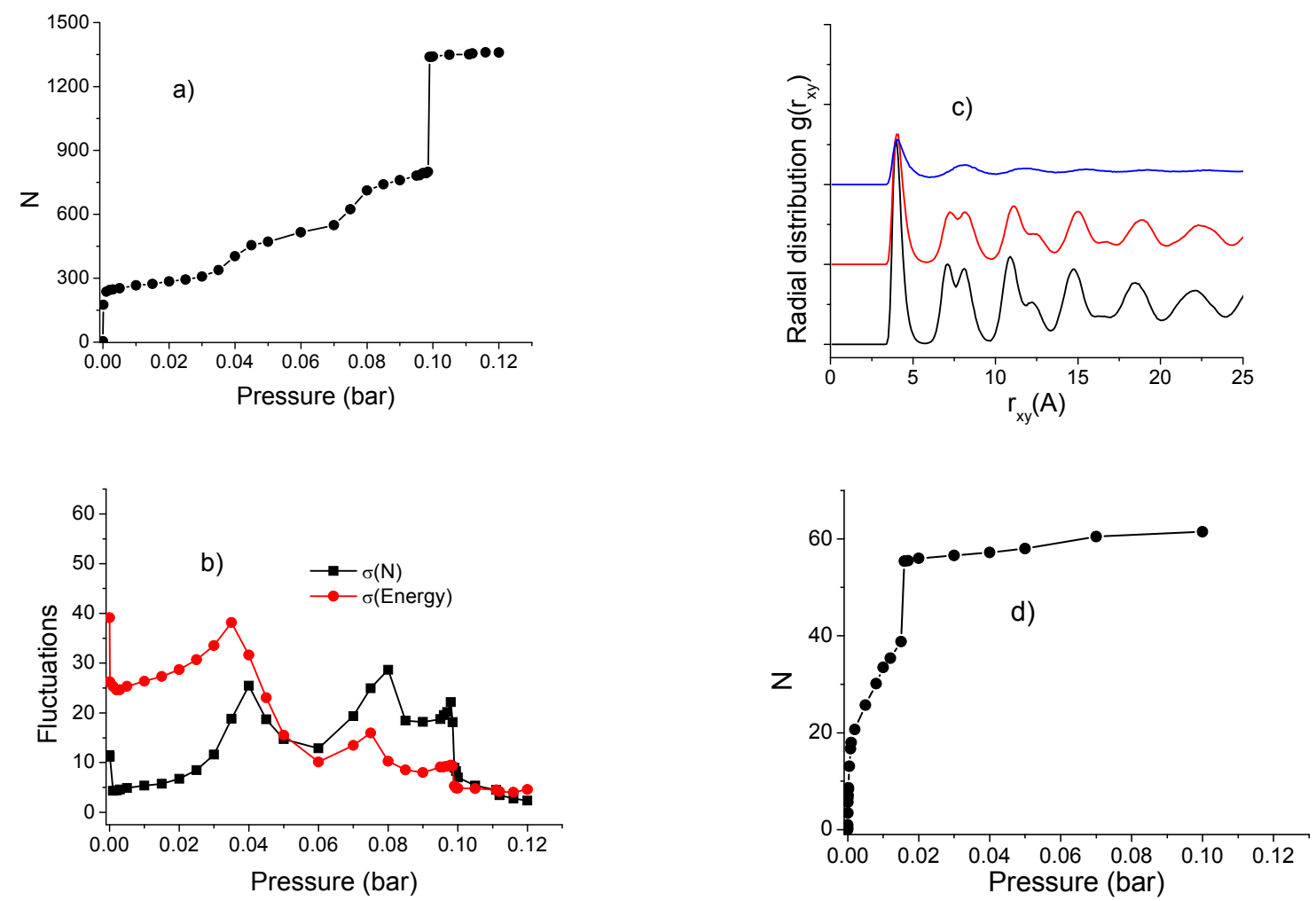

Fig. 2. Methane adsorption in a slit pore and in SURMOF (see Fig.1) at $90 \mathrm{~K}$ : (a) methane stepwise isotherm, showing subsequent layers formation in slit pores, (b) fluctuations (standard deviations from the average values) of energy and the number of adsorbed molecules in slit pores, (c) in-layer radial distribution functions: $p=0.09$ bar (first 3 layers adsorbed on each wall in $3 \mathrm{~nm}$ slit pore), (d) methane adsorption in the SURMOF pore.

The adsorption mechanism is modified when the pore geometry changes from slit-shaped to square like. Although the dimensions of square channels in SURMOF $(\mathrm{x}=\mathrm{y}=2.85 \mathrm{~nm})$ are close to the $3 \mathrm{~nm}$ slit pore, the change of pore geometry results in the increase of the energy of adsorption, due to the cumulative contributions coming from all walls. This leads to filling of the pore at pressures lower than in the slit pore: at the same thermodynamic conditions $(\mathrm{T}=90 \mathrm{~K}$, pressure $=0.002 \mathrm{bar})$ which in a slit pore at $90 \mathrm{~K}$ allows the adsorption of only the first layer, SURMOF pore is completely filled (Fig. 2d). The mechanism of methane adsorption in SURMOF is also different: (i) the adsorption of the first layer of atoms is rapid, (ii) the second layer adsorb more gradually, and (iii) filling of the central part of the pore rapidly follows the formation of the second layer. Globally, the density of the methane adsorbed in SURMOF is higher than its density in the slit pores. This is a consequence 
of stronger attractive interaction in the tetragonal pore as compared with the slit one. It is worth remembering that it happens at much lower pressure of the external gas than the filling of the slit pores. Therefore, the thermodynamic path in this case is different from the one used in the slit pores.
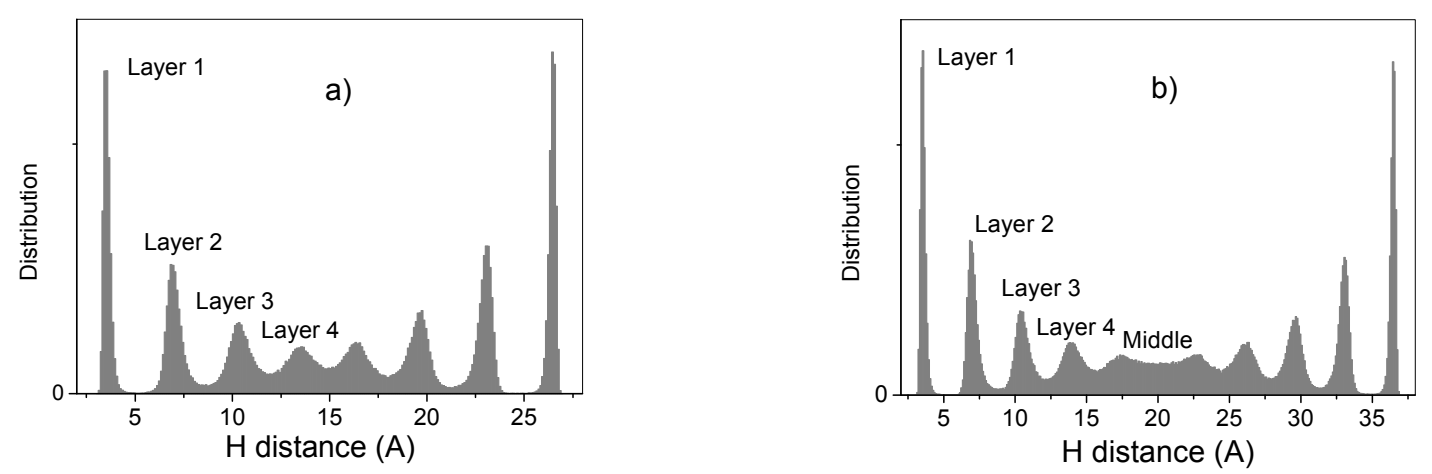

Fig. 3. (a) Mass density distribution in pore $\mathrm{z}=3 \mathrm{~nm}, \mathrm{p}=0.11$ bar; (b) mass density distribution in pore $\mathrm{z}=4 \mathrm{~nm}, \mathrm{p}=0.11$ bar, $\mathrm{T}=90 \mathrm{~K}$.

\section{B. Melting of methane confined in $3 \mathrm{~nm}$ and $4 \mathrm{~nm}$ slit pores}

At low temperatures $(\mathrm{T}<90 \mathrm{~K})$ methane adsorbed in slit-shaped pores has a well-defined layered structure (Fig. 3). However, the layers in the middle of the pore show different properties depending on the pore size. The $3 \mathrm{~nm}$ filled pore contains four well defined layer adsorbed on each pore wall (Fig. 3a). In the pore $4 \mathrm{~nm}$ wide, a fluid-like structure in the central part of the pore does not show localized layer distribution (Fig. 3b). In both systems, the first two layers are ordered, with center of molecules' mass forming a hexagonal pattern; therefore, their state can be qualified as solid. This is confirmed by calculation of the $\Phi_{6}$ ordered parameter (close to 1) and the solid-like character of the radial distribution function g(r) (Figs. 4 and 2c, respectively). 

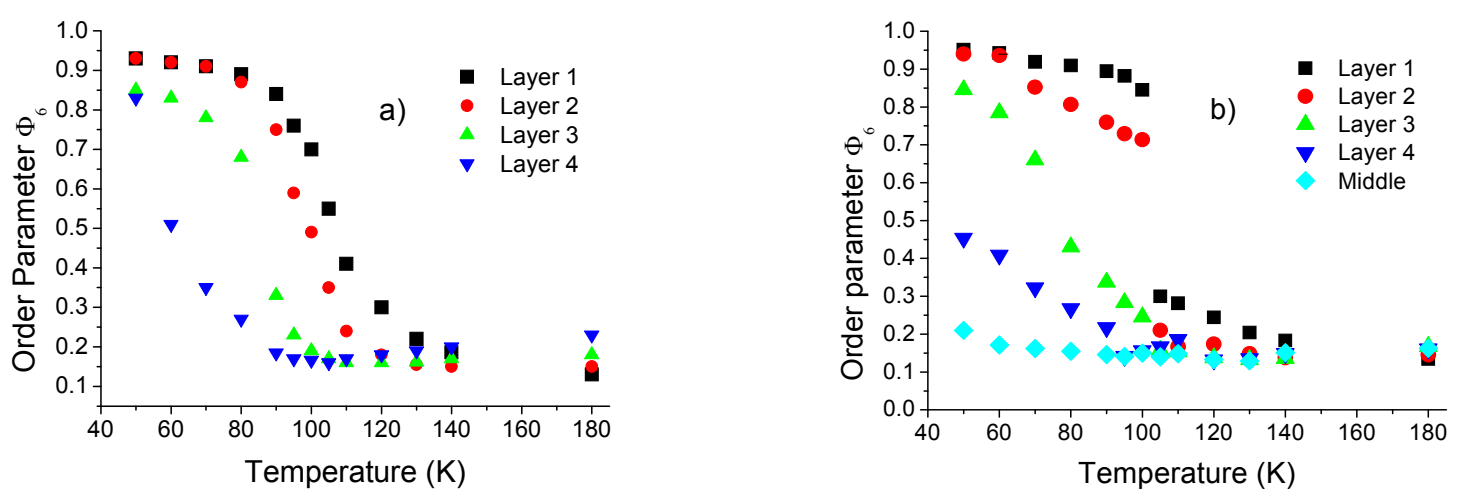

Fig. 4. Melting in slit-shaped pores: left : $z=3 \mathrm{~nm}$, right : $\mathrm{z}=4 \mathrm{~nm}$. The $\Phi_{6}$ order parameter is calculated and traced separately for each layer.

These similar nano-metric systems show important differences in the way they melt (Fig. 4). In both $3 \mathrm{~nm}$ and $4 \mathrm{~nm}$ pores, the central part of the pore has lower melting temperatures. This general feature is a consequence of the interaction of methane molecules with the confining pore walls. It induces an additional ordering of molecules in layers closer to the pore wall and, at the same time, the wall interaction is not affecting the central part of the pore. In $3 \mathrm{~nm}$ pores, all layers melt continuously. The melting of the second layer is shifted by $-5 \mathrm{~K}$ (towards lower temperature) with respect to contact layer. In $4 \mathrm{~nm}$ pores, the contact layer and the second layer exhibit a discontinuous transformation, characteristic for 3D bulk systems; their melting temperature $(\sim 105 \mathrm{~K})$ is close one to each other and higher than the bulk melting temperature $(\sim 90 \mathrm{~K})$. The difference comes partially from our choice of the melting thermodynamic path. The $3 \mathrm{~nm}$ pore is filled at lower pressure than the $4 \mathrm{~nm}$ one. Consequently, the density in the $4 \mathrm{~nm}$ pore is a little bit bigger and this leads to the discontinuous melting of the two contact layers.

The mechanism of melting in the slit pores is determined by several parameters. First, the character of transformation is strongly dependent on the strength of wall attraction as analyzed above, and pointed out in the past $^{8,9,19}$. The gradient of the attractive forces effectively acting on subsequent layers in the pores makes the adsorbed system heterogeneous and, in consequence, the mechanism of melting depends on the layer position. Additionally, the melting mechanism depends on the thermodynamic path chosen for the transformation. As the density of the system varies between different paths, the choice of the path may affect the apparent mechanism of melting. Any path between the bulk coexistence curves and the capillary coexistence curve could be used ${ }^{3}$. Here we have chosen arbitrary a path following the line between those two limits. 


\section{Melting of methane confined in SURMOF $2.8 \mathrm{~nm}$ channels}

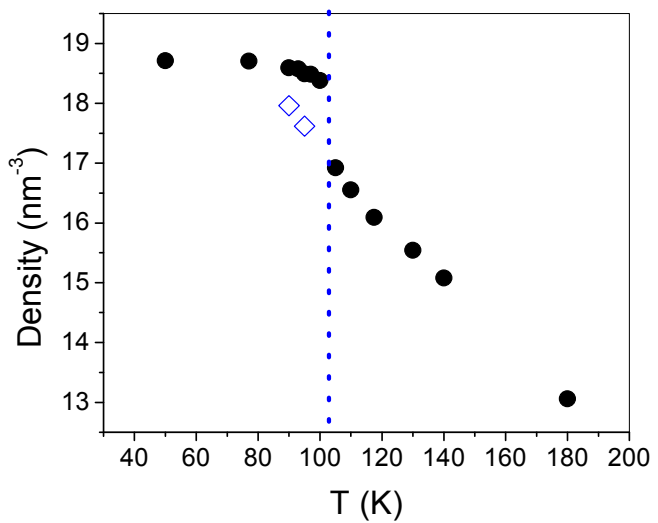

Fig. 5. Melting of methane in SURMOF. Average methane density as a function of temperature. The open symbol show hysteresis on cooling the melted system.

Below $100 \mathrm{~K}$ the structure of methane adsorbed in SURMOF pores is solid. The order in the xy planes is defined by P4 tetragonal symmetry (Fig. 6), whereas in xz and yz planes the order is hexagonal. The arrangement is not perfect, due to the system nano-metric size in xy plans and non-commensurability between the SURMOF framework and methane structure. This leads to minor deformations of the adsorbed structure, clearly observed in the density distribution shown in the Fig. 6 . The average density distribution is nearly constant below melting temperature (estimated to be $T_{\mathrm{m}}=102 \pm 2 \mathrm{~K}$ ). The constant density is a consequence of the ordered, solid-like structure, which does not change until the methane melts. However, contrary to the melting of bulk methane, the transitions in confined system is gradual. After $10 \%$ abrupt decrease of density at melting point, it then continues to decrease continuously from 17 molecules $/ \mathrm{nm}^{3}$ to 13 molecules $/ \mathrm{nm}^{3}$ by $180 \mathrm{~K}$.
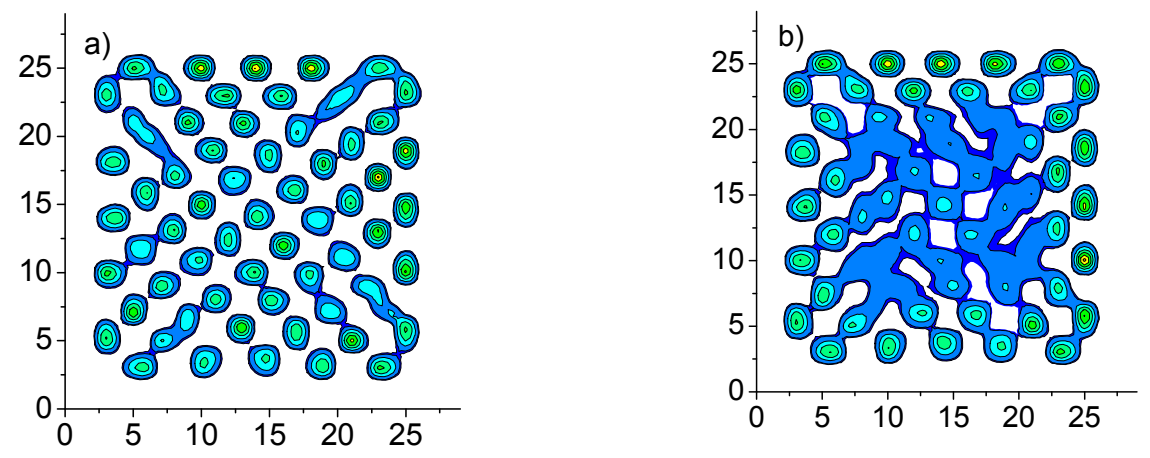

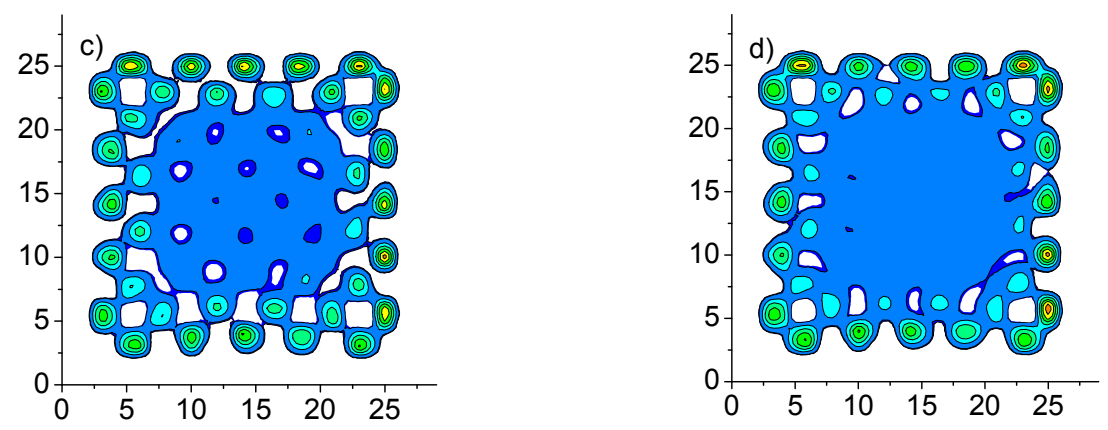

Fig. 6. Melting of methane in SURMOF. Distribution of methane density in SURMOF pore. From left to right: $90 \mathrm{~K}, 105 \mathrm{~K}$, $140 \mathrm{~K}, 180 \mathrm{~K}$. The estimated melting temperature is $102 \mathrm{~K}$.

The interaction of methane with the SURMOF framework determines the mechanism of melting. The gradual melting is a consequence of the nano-size of the pores in xy plane. The contact layers of methane are strongly localized by the framework corrugation up to the highest simulated temperature $\mathrm{T}=180 \mathrm{~K}$. Their density is unchanged up to $180 \mathrm{~K}$, the highest simulated temperature. At the same time, the inside pore fluctuations of molecules increase with temperature (Fig.6) and the density is lower towards the center of the pore. Consequently, the melted structure is strongly heterogeneous; the inside of the pore is liquid but the molecules in the contact layers stay in a solid-like state. Calculations of the $\Phi_{6}$ order parameter confirms this strong structural heterogeneity.

Obviously, it is the methane-framework interaction, which stabilizes this solid-like structure in a similar way as the first adsorbed layer in the slit-shaped pores is stabilized by methane-carbon interaction. The heterogeneous corrugation of the wall is the additional factor, which localizes the atoms in solid-like arrangement, because it limits the lateral fluctuations of the methane molecules.

\section{CONCLUSIONS}

The mechanism of melting of fluid nanosystems depends on their size, shape and type of environment in which they are embedded. The nanopores define a particular class of environments in which the molecules of the adsorbed fluid interact strongly with the porous framework; in consequence, a large variety of fluid nanosystems can be formed inside the pores, with various geometries, and length scales comparable to the characteristic scales of the intermolecular interactions. The mechanism of melting in such structures is strongly influenced by the competition between the effect of the nanometric size (which lowers the melting temperature) and the interaction of the fluid with the confining pore walls. Depending on the strength of this interaction the final transition temperature 
of the fluid nanosystems may either increase or decrease $\mathrm{e}^{8,9}$. Experimental observations ${ }^{10}$ show that in nano-pores of the width smaller than $4 \mathrm{~nm}$ the mechanism of melting is no longer similar to that observed in the bulk systems. This is a consequence of a strong heterogeneity of the adsorbed nano-fluid where each layer of adsorbed molecules shows different properties.

Specifically, the nano-structures composed of few molecular layers (therefore, having at least one dimension smaller than $4 \mathrm{~nm}$ ) are very heterogeneous. Consequently, the temperature (and the mechanism) of system phase transition is not unique and vary in a function of the position within the nano-system. In general, melting in nano-systems cannot be described by global parameters; it must be analyzed locally, depending on the molecules of fluid position and the distance from all pore walls. The density distribution is a graphical way of the melting mechanism analysis. The locally defined order parameters provide another, quantitative description of the phenomena. This approach is qualitatively different from the analysis of melting in pores larger than $4 \mathrm{~nm}$, for which the classical picture of melting point depression is typically observed ${ }^{10}$. The order parameter $\Phi_{6}$ is one of many possible ways to characterize local structures. The choice depends on the symmetry of the studied system.

In addition, the mechanism of melting in confined systems depends on the thermodynamic path chosen to transform the solid into the fluid. In pores, there are many possible choices depending on the number of adsorbed layers. The pressure may follow any path in the $(\mathrm{P}, \mathrm{T})$ plane at any stage of adsorption. In the completely filled pore, any path between the filling pressure and the saturation pressure can be chosen. In pores where the pore filling is observed well below the saturation pressure, there are many choices of the melting thermodynamic path. However, higher pressure means higher density of the confined system that, in turn, can lead to discontinuous melting and/or may produce a hysteresis. The heterogeneity of system is additional and the most important factor determining the mechanism of melting.

We have observed an evolution of the melting mechanism as a function of the pore size. It is discontinuous transformations within the contact layers in $4 \mathrm{~nm}$ slit pores to the totally continuous one in $3 \mathrm{~nm}$ pore. The type of the transformation depends on the density of the confined system, which is affected by the strength of the interactions with the walls and by the equilibrium external gas pressure. Exploring this added "dimensions" in the parameter space is very interesting but requires a significant additional effort beyond the scope of this manuscript. 


\section{SUPPLEMENTARY MATERIAL}

See supplementary material for detailed SURMOF structure (x,y,z atomic positions) used in the simulation.

\section{ACKNOLEDGEMENTS}

This work was partially supported by the Polish National Science Center (NCN, grant no. 2015/17/B/ST8/00099) and French National Research Agency ANR (grant NR-14-CE05-0009HYSTOR). CW acknowledges support from the donors of the American Chemical Society Petroleum Research Fund (52696-ND5).

\section{REFERENCES}

${ }^{1}$ D. W. Oxtoby, J. Phys. Cond. Matt. 4, 7627-7650 (1992)

${ }^{2}$ A. Maattanen, H. Vehkamaki, A. Lauri, S. Merikallio, J. Kauhanen, H. Savijarvi and M. Kulmala J. Geophys. Res. 110, E02002 (2005)

${ }^{3}$ M. Miyahara and K. E. Gubbins J. Chem. Phys. 106, 2865 (1997)

${ }^{4}$ M. W. Maddox and K. E. Gubbins, J. Chem. Phys. 107, 9659-67 (1997)

${ }^{5}$ R. Radhakrishnan and K. E. Gubbins, Molecular Physics 96, 1249-1267 (1999)

${ }^{6}$ T. X. Nguyen, S. K. Bhatia, and D. Nicholson, J. Chem. Phys.117, 10827-36 (2002)

${ }^{7}$ K. G. Ayappa and C. Ghatak, J. Chem. Phys. 117, 5373-83 (2002)

${ }^{8}$ R. Radhakrishnan, K. E. Gubbins, M. Sliwinska-Bartkowiak, J. Chem. Phys., Vol. 116, 1148-56 2002

${ }^{9} \mathrm{C}$ Alba-Simionesco, B Coasne, G Dosseh, G Dudziak, K E Gubbins, R Radhakrishnan and M

Sliwinska-Bartkowiak, J. Phys.: Condens. Matter 18, R15-R68 (2006)

${ }^{10}$ Hugo K Christenson, J. Phys.: Condens. Matter 13, R95-R133 (2001)

${ }^{11}$ Liu J, Lukose B, Shekhah O, et al. Scientific Reports. 2, (2012)

${ }^{12}$ Etters, RD; Kuchta, B; Belak, J Phys. Rev. lett 70, 826-829 (1993)

${ }^{13}$ Kuchta, B; Luty, T. J. Chem. Phys 78, 1447-1452 (1983)

${ }^{14}$ B. Kuchta, L. Firlej, P. Pfeifer and C. Wexler, Carbon 48, 223 -231 (2010) 
${ }^{15}$ M. Golebiowska, M. Roth, L. Firlej, B. Kuchta, and C. Wexler. Carbon 50, 225-234 (2012)

${ }^{16}$ D. Nicholson and G. Parsonage, "Computer Simulation and the Statistical Mechanics of Adsorption." Academic Press, London, (1982).

${ }^{17}$ M. Razak, D.D. Do, and G.R. Birkett. Adsorption 17, 385-394 (2011)

${ }^{18}$ L.Firlej, B.Kuchta, M. W.Roth, M. J. Connolly and C. Wexler, Langmuir 24, 12392-97 (2008)

${ }^{19}$ L. Ortiz, B. Kuchta, L. Firlej, M.W. Roth and C. Wexler, Mater. Res. Express 3 (2016) 055011 


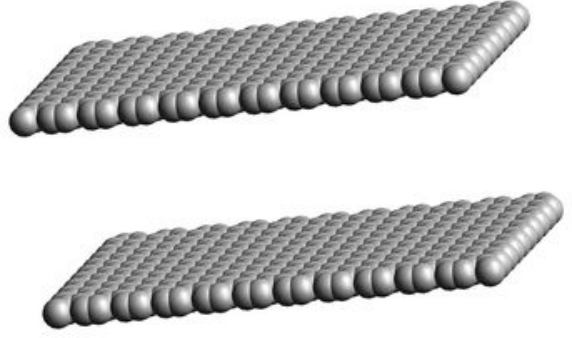





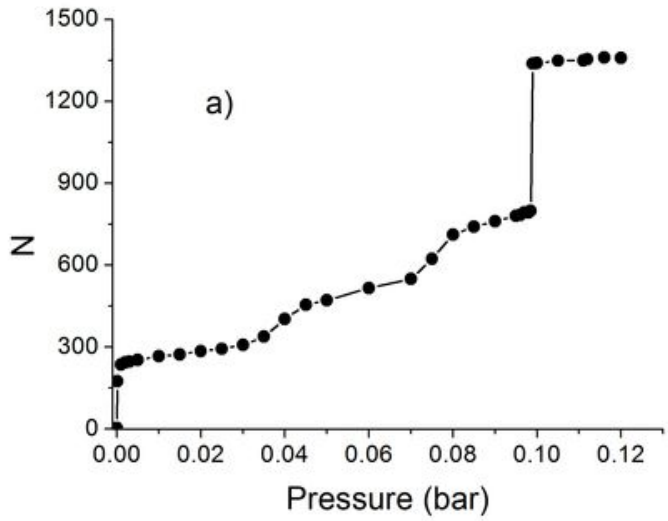




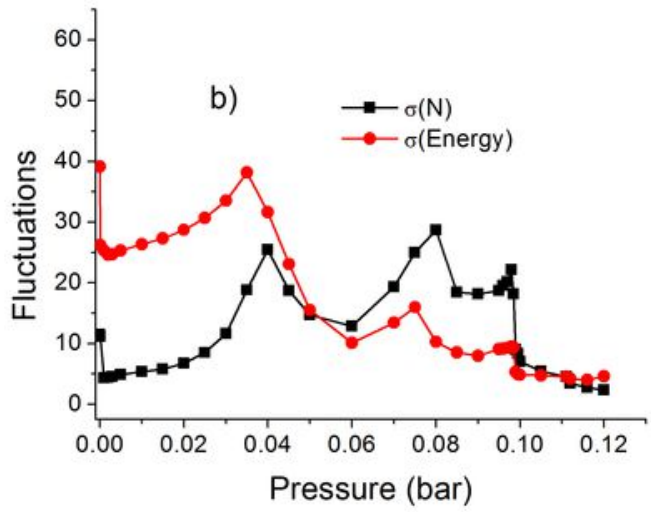




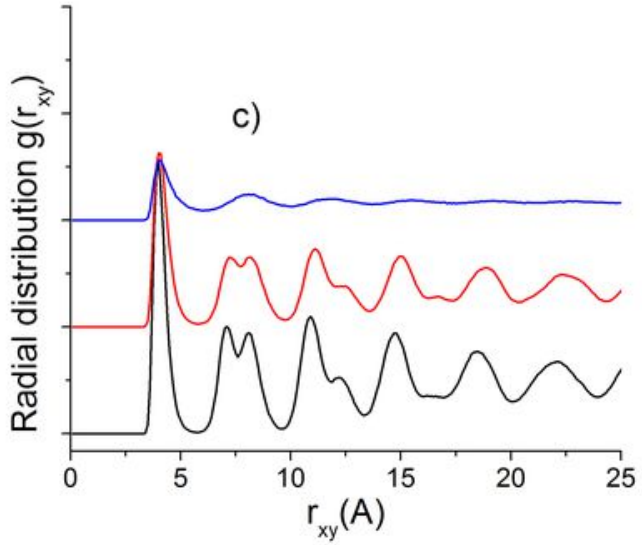




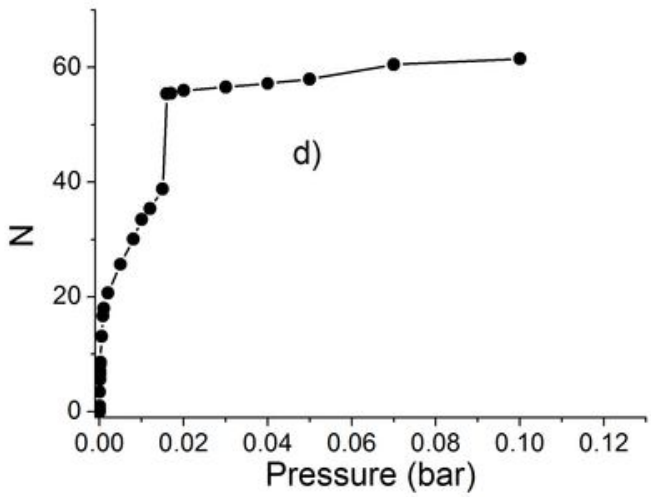




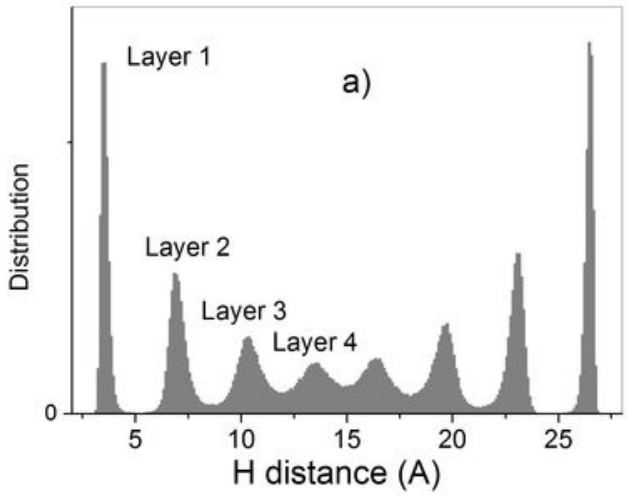




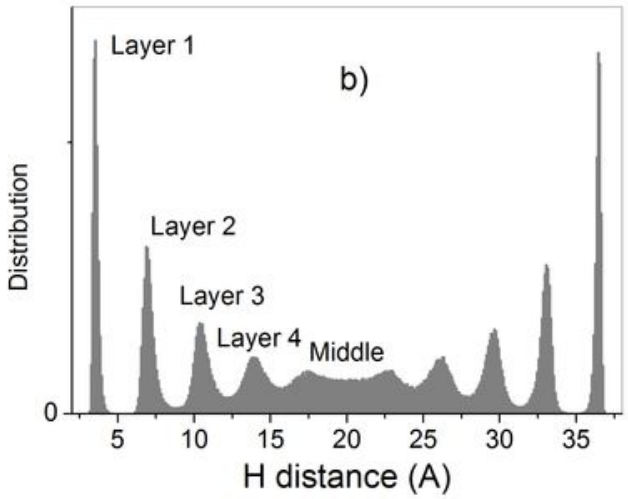




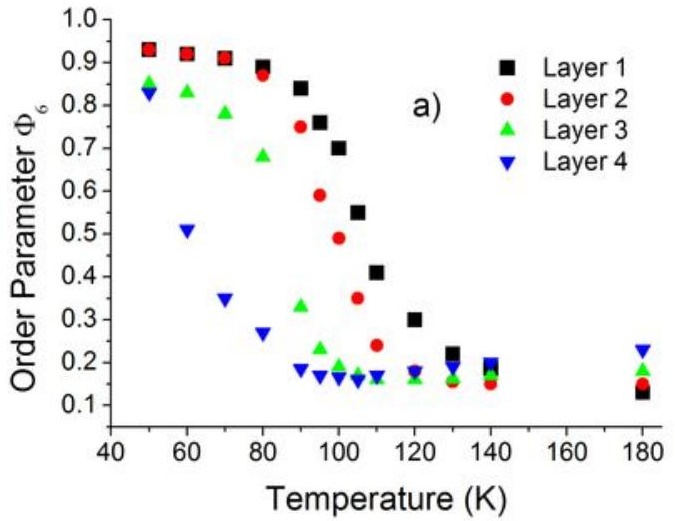




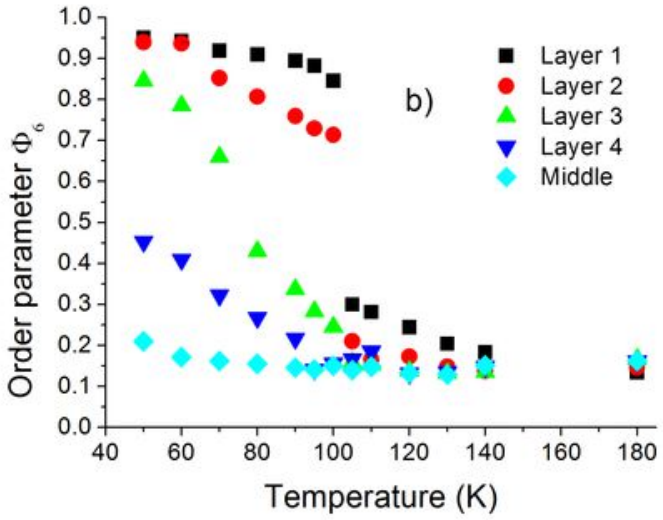




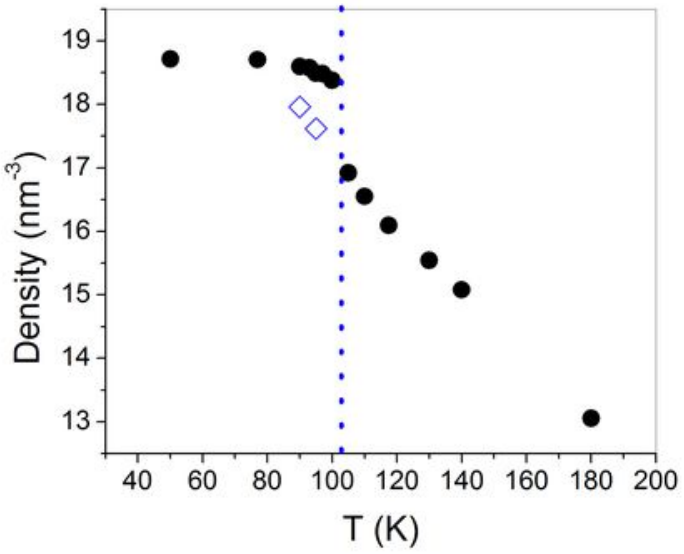




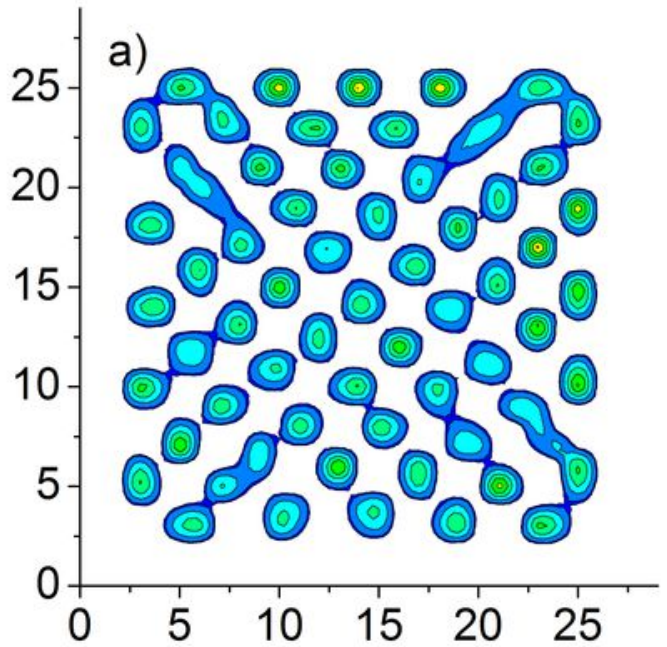




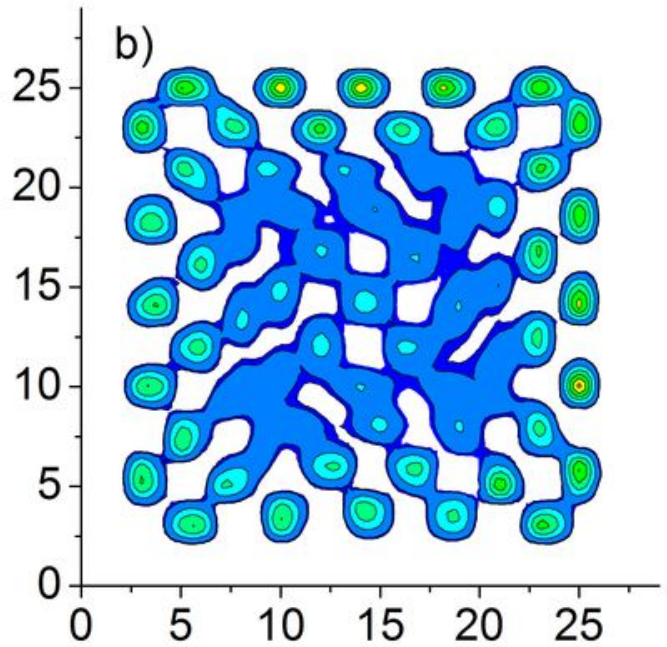




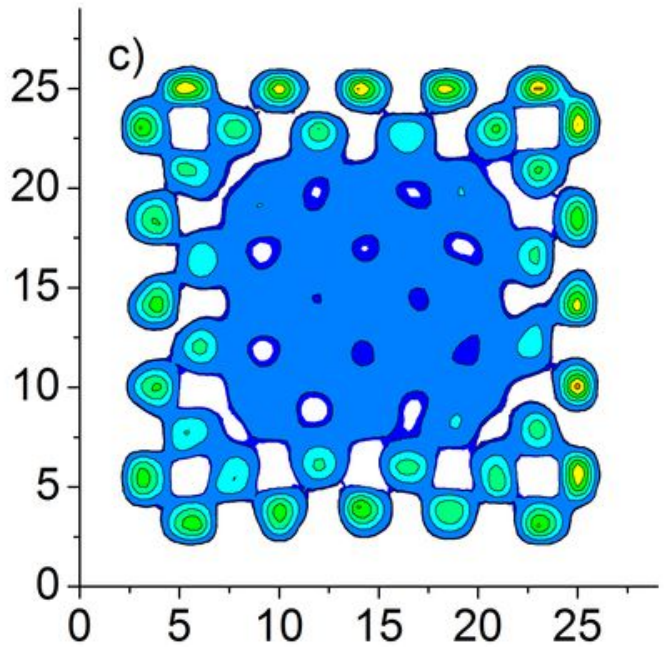




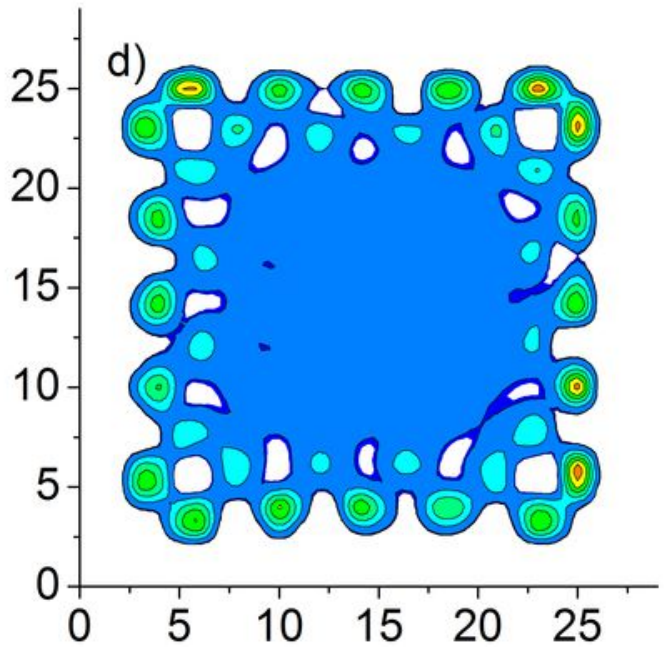

